\title{
Depression in the elderly with visual impairment and its association with quality of life
}

\author{
This article was published in the following Dove Press journal: \\ Clinical Interventions in Aging \\ 18 July 2013 \\ Number of times this article has been viewed
}

\author{
Judith Renaud \\ Emmanuelle Bédard \\ School of Optometry, University of \\ Montreal, Montreal, Quebec, Canada
}

Correspondence: Judith Renaud

École d'optométrie, Université de

Montréal, 3744 Jean-Brillant local 260-7,

Montréal, Québec,

H3T IPI, Canada

$\mathrm{Tel}+\mathrm{I} 5$ I4 34375 I3

Fax +I 5 |43432382

Email judith.renaud@umontreal.ca
Background: Visual impairment is more prevalent in the elderly and depression is common in this population. Although many studies have investigated depression or quality of life (QOL) in older adults with visual impairment, few have looked at the association between these two concepts for this population. The aim of this systematized review was to describe the association between depression and QOL in older adults with visual impairment.

Methods: A search was done using multiple electronic databases for studies addressing the relationship between QOL and depression in elders with visual impairment. The concept of QOL was divided into two different approaches, ie, QOL as achievement and QOL as subjective well-being. Comparison of QOL scores between participants with and without depression (Cohen's $d$ ) and correlations between depression and QOL (Pearson's $r$ ) were examined.

Results: Thirteen studies reported in 18 articles were included in the review. Nearly all of the studies revealed that better QOL was moderately to strongly correlated with less severe depressive symptoms ( $r=0.22-0.68$ for QOL as achievement; $r=0.68$ and 0.72 for QOL as subjective well-being). Effect sizes for the QOL differences between the groups with and without depression ranged from small to large ( $d=0.17$ to 0.95 for QOL as achievement; no data for QOL as subjective well-being).

Conclusion: Additional studies are necessary to pinpoint further the determinants and mediators of this relationship. Considering the high prevalence rate of depression in this community and its disabling effects on QOL, interventions to prevent and treat depression are essential. More efforts are needed in clinical settings to train health care practitioners to identify depressed elders with visual impairment and provide appropriate treatment.

Keywords: depressive symptoms, disability, health-related quality of life, vision-related quality of life, subjective well-being, older adults

\section{Introduction}

The prevalence of vision loss in the elderly is high, being about $15 \%$ for people aged 65 years and older and up to $30 \%$ in people 75 years and over. ${ }^{1-4}$ The onset of visual impairment in later life alters life habits and has various consequences. For example, older people with visual impairment present more restrictions in participation than their peers, ${ }^{5,6}$ have less social interaction, ${ }^{1,7-9}$ feel lonelier, ${ }^{10,11}$ and are at risk of developing depressive symptoms. ${ }^{12-15}$ In the literature, there is strong evidence that visual impairment is related to depression. Consideration should be given to how depression affects the quality of life (QOL) of older adults with visual impairment.

Historically, the success of health interventions has been evaluated from an outsider perspective, using tests focusing on observable life conditions or physical functioning. 
Over the last two decades, evaluation of outcomes by the patient, giving the insider perspective, has become increasingly popular. In this new paradigm, it is important to assess QOL when evaluating health research and interventions.

Despite its popularity, there is still no consensus about the conceptualization of QOL, apart from an agreement that it is multidimensional, personal, should primarily be evaluated subjectively, and can vary over time. Some authors consider that this concept incorporates two major approaches, ie, QOL as achievement and QOL as subjective well-being. ${ }^{16-18} \mathrm{QOL}$ as achievement refers principally to the person's functional status. It mainly assesses the capacity, as perceived by the individual him/herself, to do a particular activity, such as reading, dressing, or walking, as well as to take care of basic needs to stay healthy and assume social roles. Usually, the tools used to evaluate this aspect of QOL do not allow for any modulation based on the person's perspective or goals. Health-related QOL is a major category of QOL as achievement. QOL as subjective well-being, which will be referred to as subjective QOL in this paper, represents mostly the sum of the cognitive and emotional reactions describing the person's satisfaction with life and well-being in relation to his/her goals, expectations, concerns, values, and priorities. Subjective QOL evaluation depends mainly on the congruence or discrepancy between the person's achievements and expectations. It tends to reflect a more global aspect of QOL. The instruments used to assess subjective QOL aim to understand the way individuals perceive their condition according to their values and expectations.

QOL can be seriously affected by vision loss. In fact, visual impairment is a very disabling condition, especially when it is acquired in later life. ${ }^{19-21} \mathrm{~A}$ study by Brown et al found that severe age-related macular degeneration affects QOL in a way that is comparable with advanced prostate cancer, with unmanageable pain along with bladder and sexual dysfunction, or with a severe stroke leading to constant nursing care, incontinence, and paralysis. ${ }^{22}$ The burden of vision loss is substantial and its consequences are numerous, from functional to social and psychological, and it can lead to depression. ${ }^{23,24}$ The prevalence of major depression in older adults with visual impairment is estimated to be around $14 \%,{ }^{25}$ while depressive symptoms affect about one third of elders with visual impairment. ${ }^{15,24,26}$ In turn, depression affects elders' QOL. ${ }^{27}$

Previous studies have shown that older adults with clinically significant depressive symptoms have worse health-related QOL and greater disability over time. ${ }^{28}$ Characteristics of depression include aversion to activities previously enjoyed, lack of stamina, and poor concentration. ${ }^{29}$ Depressive symptoms therefore contribute to the "disablement process speeding up disease to disability". ${ }^{30}$ This is particularly true with visual impairment, since daily tasks require a great deal of concentration and effort.

Even though medicine has made important advances in the treatment of age-related eye diseases in recent years, many patients still suffer from noncorrectable vision loss. ${ }^{21}$ With an aging population in developed countries, the number of older people with visual disabilities is expected to increase substantially in the years to come. ${ }^{31,32}$ Knowing that visual impairment is more prevalent in the elderly and that depression is common in this population, it is important to look at the impact of depressive symptoms on older people's QOL. Although many studies have investigated depression or QOL of older adults with visual impairment, few have looked at the association between these two concepts for this population. The aim of this systematized review was to describe the association between depression and QOL in older adults with visual impairment.

\section{Materials and methods Literature search strategy}

A search of the literature was done using multiple databases, ie, MEDLINE, PsychInfo, Pubmed, EMBASE, Social Work Abstracts, and Cochrane. An attempt was made to find gray literature via Google Scholar and proceedings of international scientific meetings. Bibliographies of articles were searched manually for additional studies. The terms mapped were visual conditions such as "visual impairment", "macular degeneration", or "glaucoma" associated with depression terms such as "depressive disorder" or "depressive symptoms" and QOL terms such as "life satisfaction", "well-being", or "quality of life". The research was limited to elders and studies were manually sorted to keep only those with older participants ( $>55$ years) having visual impairment. Visual impairment was defined as any eye disease causing a noncorrectable decrease in vision that corresponds to a visual acuity of 6/12 (20/40) or less or a visual field of $60^{\circ}$ or less in the better eye. Self-reported visual impairment was included if participants were receiving services from a vision rehabilitation agency. All types of study design were included except case reports. Reviews and meta-analyses were not analyzed but were used to find more relevant articles. The search was limited to literature from 1980 to June 1, 2012 in English or French. EndNote X5 (Thomson Reuters X5.0.1, Carlsbad, CA, USA) was used to manage the database. 
A total of 339 articles were found using the database search. This number was reduced to 18 after reading the abstracts or complete articles (see Figure 1). Most studies were removed because the analysis did not look at the association between depression and quality of life, or the majority of the sample did not suffer from visual impairment. Questionnaires had to assess QOL as perceived by the individual (patient-reported outcomes).

\section{Evaluation of methodological quality of studies}

There are numerous tools available to assess the methodological quality of studies.$^{33}$ Critical elements considered (eg, susceptibility to bias, confounding as well as the number and weighting of items) can vary widely among tools. Thus, evaluation of a study with different tools can result in different levels of quality. We choose the Cho and Bero scale because it allows assessment of experimental as well as observational studies. ${ }^{34}$ Also, its development has been documented, and its validity and reliability have been previously tested. ${ }^{34}$ Both authors read all the articles and independently assessed the methodological quality of each study using this 24 -item scale. The score was calculated as a fraction of the total score out of the number of items applicable. Thus, the total score varied between 0 and 1 , with higher scores indicating better methodological quality. For the purpose of the analysis, the calculated score was used to qualify a study as excellent (score $>0.85)$, good $(0.70-0.85$ ), fair $(0.55-0.69)$, or poor $(<0.55)$. Studies where there were discrepancies between the reviewers' scores were re-evaluated and discussed to decide the final score. The score for the studies included in the review fluctuated between 0.62 (fair) and 0.77 (good); none achieved a perfect score, mainly because of study design, measurement bias, or confidence intervals not being reported. Studies with very poor methodological quality (Cho score $<0.35$ ) were excluded.

Studies were classified according to the study level. The classification used was as follows: randomized controlled trial (I), cohort (II), case control (III), and case series (IV). ${ }^{35}$

\section{Data extraction}

Characteristics of the population, study design, type of questionnaire, and relevant outcomes were extracted. Data were collected systematically for every correlation (Pearson's $r$ ), odds ratio and variance related to the association between

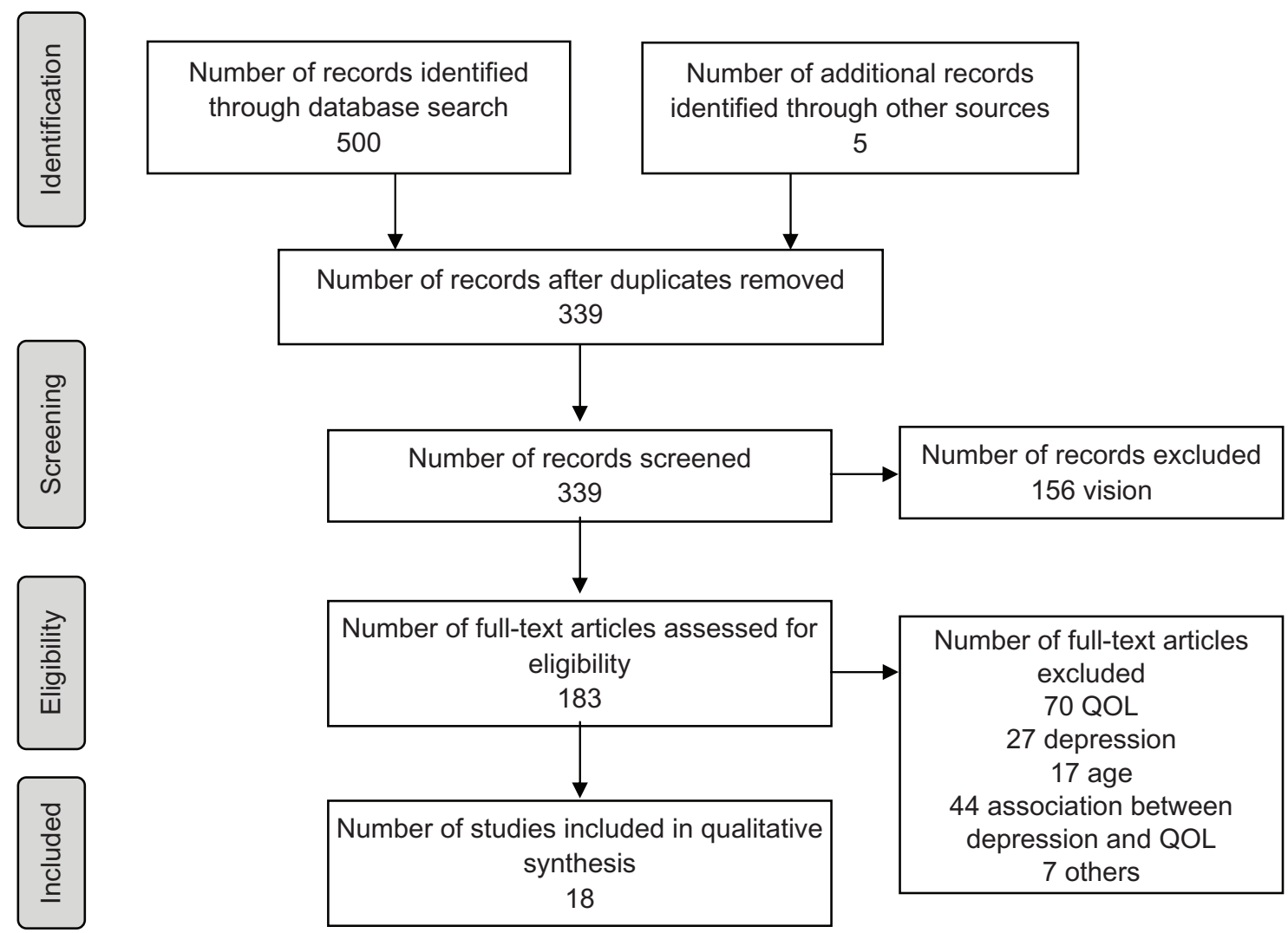

Figure I PRISMA flow chart.

From: Moher D, Liberati A, Tetzlaff J, Altman DG, The PRISMA Group (2009). Preferred Reporting Items for Systematic Reviews and Meta-Analyses: The PRISMA Statement. PLoS Med 6(6): el000097. doi: 10.137I/journal.pmed I000097.

Abbreviation: QOL, quality of life. 
depression and QOL, QOL comparison data between groups with and without depression (mean and standard deviation), and prevalence of depression. The strength of a correlation was assessed using Cohen's scale ${ }^{36}$ in which a correlation, in absolute value, of $\geq 0.5$ was qualified as a strong association, from 0.3 to 0.49 as moderate, from 0.1 to 0.29 as weak, and $\leq 0.09$ as no correlation. For the comparison between two means concerning QOL, Cohen's $d$ was calculated to give a standardized mean effect size when the mean and standard deviation of the group with depressive symptoms and the group without depressive symptoms were provided. A $d=0.2$, in absolute value, was considered to be a small effect size, 0.5 a medium effect size, and 0.8 a large effect size. ${ }^{36}$ No meta-analysis was conducted because the studies were too dissimilar, among other things, in methodology, measuring tools, or accounting for confounding factors.

After reading the papers in detail, we realized that some described the same sample and were based on the same project. After discussion, we agreed to aggregate the results of these multiple papers depicting the same project and consider them as a single study. ${ }^{15,20,24,37-43}$ Therefore, 13 studies (11 observational and two interventional) reported in 18 papers were included in this review.

\section{Results}

\section{Studies included in the review}

Thirteen studies (18 articles) addressing the relationship between depression and QOL were selected. Five of the studies were cross-sectional (IV), one was a case-control (III) study, and seven were follow-up studies (II). There were no randomized clinical trials (I). The follow-up time ranged from six to 48 months. Most of the studies originated in the United States $(n=9)$ while the others were conducted in Australia $(n=1)$, Canada $(n=1)$, New Zealand $(n=1)$, or the United Kingdom $(n=1)$. Eight of the American studies were generated by two different research groups: one team headed by Horowitz and Reinhardt was from a vision rehabilitation agency in New York, and the other group directed by Rovner was based at an ophthalmological hospital in Philadelphia. Although the focus of most of the selected studies was not the association between depressive symptoms and QOL, Rovner et al were directly interested in the effect of depression on functional disability (health-related and vision-related QOL).

The study populations came primarily from low vision rehabilitation agencies or specialized ophthalmology clinics. The sample size varied greatly, ranging from 31 to 438 participants. For the majority, the population was very old, with a mean age varying from 77 to 84 years. The visual impairment was recent in most of the studies and was the result of age-related eye diseases without any distinction (eight studies) or was limited to one disease in particular, more specifically age-related macular degeneration (five studies).

Five different instruments were used to measure depressive symptoms (see Table 1); the prevalence of significant depressive symptomatology ranged from $29.4 \%$ to $43.4 \%{ }^{13,14,20,24,39,40,42,44}$ (see Table 1). Three studies assessed depressive disorders by clinical interview according to the Diagnostic and Statistical Manual of Mental Disorders (DSM) criteria or using the Structured Clinical Interview for DSM-IV (SCID-IV); the prevalence varied between $7.2 \%$ and $38.6 \% .{ }^{15,38,45}$ Although half of the studies assessed the relationship between depression and both health-related and vision-related QOL $(\mathrm{n}=7)$, some investigated only the association with either health-related $(n=2)$ or vision-related $(\mathrm{n}=4)$ QOL. Only two studies looked at the association between depression and subjective QOL.

\section{Outcomes of interest}

To evaluate the relationship between depression and QOL, we focused on two main elements, ie, QOL comparisons between participants with and without depressive symptoms (mean \pm standard deviation, Cohen's $d$ ), and correlations between depression and QOL (Pearson's $r$ ) (see Table 2). The outcomes are discussed in three main sections, ie, depression and health-related QOL, depression and vision-related QOL, and depression and subjective QOL. The first section, health-related QOL, includes generic instruments measuring essentially the person's functional limitations related to health status. The second section, vision-related QOL, includes vision-specific tools. These questionnaires assessing mainly difficulty in vision-related activities aim to determine the impact of vision loss on QOL as perceived by the individual. Health-related and vision-related QOL are both included in QOL as achievement. The third section presents the studies which assessed subjective QOL.

\section{Depression and health-related QOL}

In the studies included in this review, four different tools were used to assess general health-related QOL (number of studies): Community Disability $\operatorname{Scale}^{46}(\mathrm{n}=2)$, Medical Outcomes Study Short-Form Health Survey ${ }^{47}$ (SF-36, $\mathrm{n}=2$ ), Older Americans Resources and Services Multidimensional Functional Assessment Questionnaire ${ }^{48}(n=4)$, and Sickness Impact Profile ${ }^{49}(n=1)$. 
Table I Prevalence of depression in the selected studies

\begin{tabular}{|c|c|c|c|c|c|}
\hline Clinical interview & Reference & Year & Major depression (\%) & $\mathbf{n}$ & Population \\
\hline DSM-III-R criteria & Shmuely-Dulitzki et al ${ }^{38}$ & 1995 & 38.6 & 70 & $\mathrm{VI}$ \\
\hline \multirow[t]{2}{*}{ SCID-IV } & Brody et $\mathrm{a}^{45}$ & 2001 & $\begin{array}{l}32.5 \text { (depressive } \\
\text { disorders) }\end{array}$ & $15 \mid$ & AMD \\
\hline & Horowitz et al ${ }^{15}$ & 2005 & 7.2 & 584 & VI \\
\hline Questionnaire & Reference & Year & DS (\%) & $\mathbf{n}$ & Population \\
\hline \multirow[t]{4}{*}{$\overline{C E S-D}$} & Horowitz et $\mathrm{al}^{39,40}$ & 2003 & 33.7 & 155 & VI \\
\hline & & 2005 & $\begin{array}{l}25.3 \text { (two-year post } \\
\text { intervention) }\end{array}$ & 95 & \\
\hline & Reinhardt et $\mathrm{a}^{20}$ & 2001 & 35 & 570 & VI \\
\hline & Rovner et al ${ }^{42}$ & 2002 & 33.3 & 51 & AMD \\
\hline GAD & Mathew et $\mathrm{al}^{13}$ & 2011 & $43.4(17.3)$ & $145(104)$ & AMD (control) \\
\hline \multirow[t]{3}{*}{ GDS } & Rovner et $\mathrm{al}^{24}$ & 1996 & 38.7 & 31 & $\mathrm{VI}$ \\
\hline & Hayman et $\mathrm{al}^{44}$ & 2007 & 29.4 & 391 & VI \\
\hline & Renaud et $\mathrm{al}^{14}$ & 2010 & 31 & 64 & VI \\
\hline HDRS & Rovner et $\mathrm{al}^{56}$ & 2006 & 23.8 (minimal DS) & 206 & AMD \\
\hline $\mathrm{PHQ}$ & Rovner et $\mathrm{al}^{57}$ & 2011 & $\begin{array}{l}\text { I2.9 (depressive } \\
\text { disorders) }\end{array}$ & 241 & AMD \\
\hline
\end{tabular}

Abbreviations: AMD, age-related macular degeneration; CES-D, Center for Epidemiological Studies-Depression Scale; DSM-III-R, Diagnostic and Statistical Manual of Mental Disorders, 3rd Edition, Revised; DS, depressive symptoms; GAD, Goldberg Anxiety and Depression scale; GDS, Geriatric Depression Scale; HDRS, Hamilton Depression Rating Scale; PHQ, Patient Health Questionnaire; VI, visual impairment; SCID-IV, Structured Clinical Interview for the Diagnostic and Statistical Manual of Mental Disorders, 4th Edition.

Two studies by Reinhardt et al using the Older Americans Resources and Services Multidimensional Functional Assessment Questionnaire showed a significant moderate association between health-related QOL and depressive symptoms in community-dwelling older adults with visual impairment recruited at a low vision rehabilitation agency. ${ }^{37,50}$ Using the same questionnaire, in two longitudinal studies, Horowitz et al also found a weak to moderate significant association for elders with visual impairment seeking rehabilitation services. ${ }^{15,39-41}$ In their study of 438 elders with visual impairment, participants with subthreshold depression or depression had significantly lower health-related QOL than nondepressed participants (medium to large effect size).

The most widely known health-related QOL questionnaire, the SF-36, is divided into two components, physical and mental health, each including four domains. The two studies using that tool showed that more depressive symptoms were significantly related to worse health-related QOL. ${ }^{13,44}$ In a study by Mathew et al, ${ }^{13}$ the associations in all the physical health domains were moderate except for one, which was strong, while the correlations were strong for all the mental health domains. Therefore, the mental component of the SF-36 had a stronger correlation with depressive symptoms than the physical component in this Australian study. In a study by Hayman et al, participants with significant depressive symptoms presented lower scores than nondepressed participants for the different SF-36 subscales (medium effect size for the physical component, and large effect size for the mental component); the standardized difference between the two groups was larger for the mental component than for the physical component. ${ }^{44}$ Fewer depressive symptoms were also significantly related to better health-related QOL. ${ }^{44}$

The two follow-up studies by Rovner et al showed that older adults with visual impairment and significant depressive symptoms had significantly greater disability (poorer healthrelated QOL) than nondepressed elders (medium effect size) using the Community Disability Scale..$^{24,42}$ In addition, more depressive symptoms were significantly associated with greater disability, and the strength of the association varied from moderate to strong. In their study of 70 elders with visual impairment at baseline, regression analysis showed that depressive symptoms alone explained $20 \%$ of health-related QOL, while the severity of visual impairment explained an additional $7 \%{ }^{38}$ In the group of participants who completed the study $(n=31), 10$ of the 12 who initially had significant depressive symptoms remained depressed two years later; these authors concluded that depressive symptoms left untreated are persistent in this population. ${ }^{24}$

Brody et $\mathrm{a}^{45}$ used the 68 -item Sickness Impact Profile version in their study. Participants with depression had greater disability than nondepressed participants (large effect size). The results also pointed to a significant strong correlation between depressive symptoms and health-related QOL.

In sum, all the studies showed a significant association between fewer depressive symptoms and better general health-related QOL. The strength of the correlations was moderate to strong, varying from 0.23 to 0.68 , with a median of 0.43 . Four studies found a statistically significant health- 


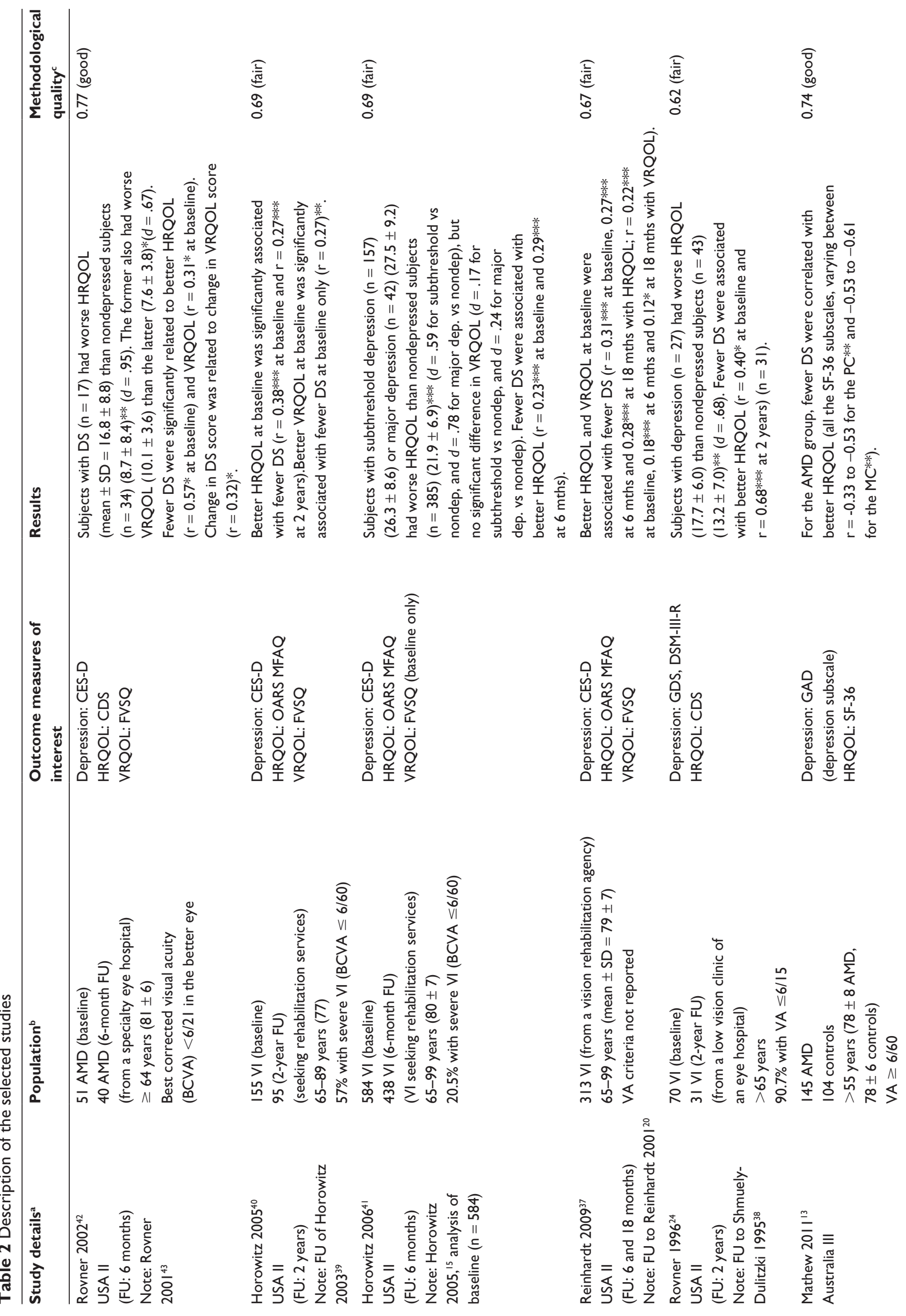




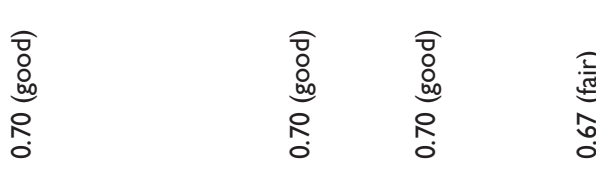

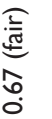

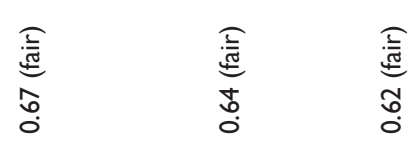

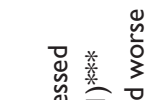

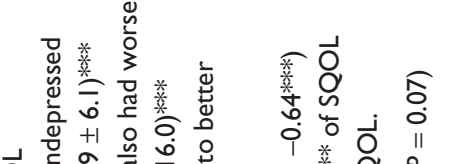

О人

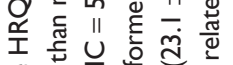

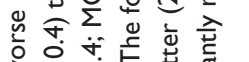

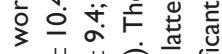

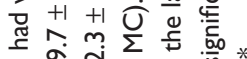

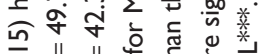

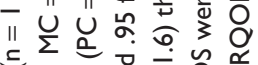

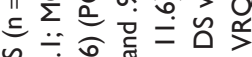

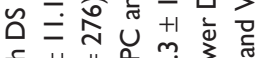

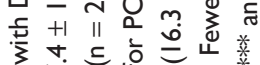

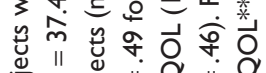

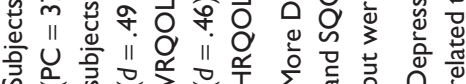

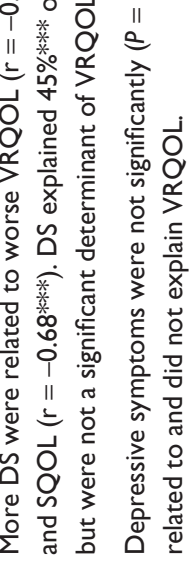

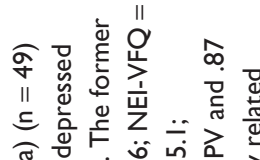

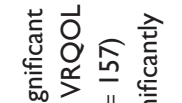

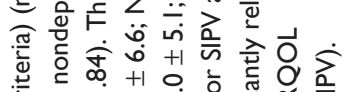

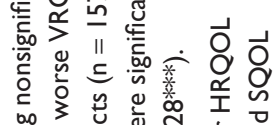

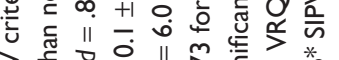

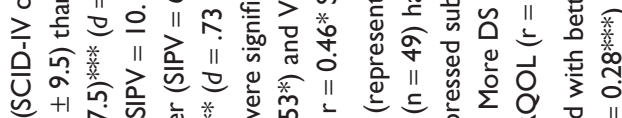

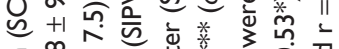

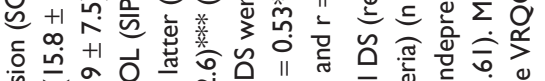

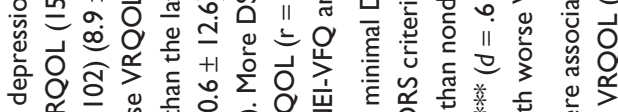

传蒫

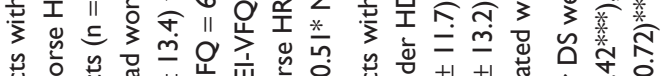

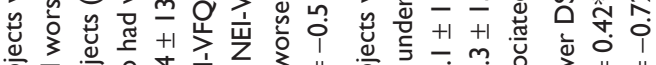

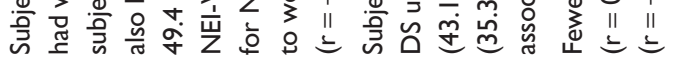
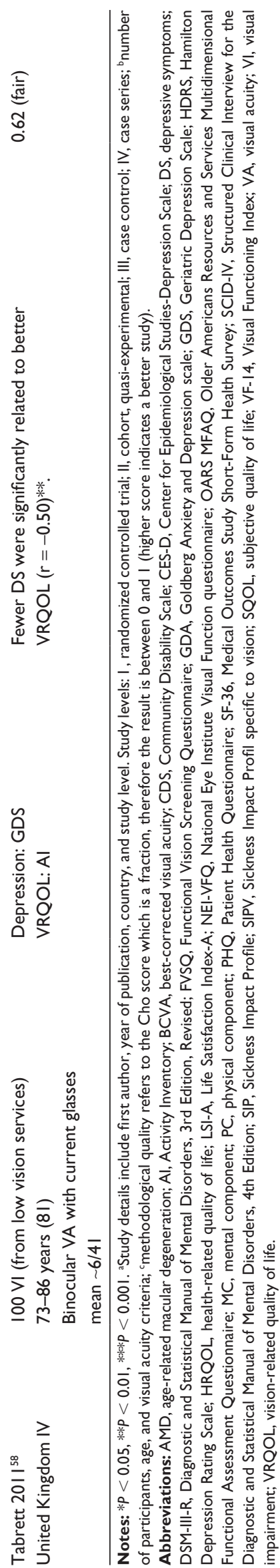

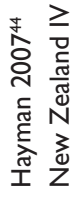

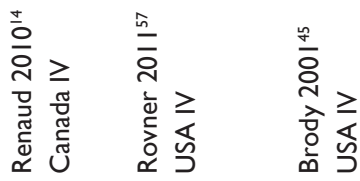
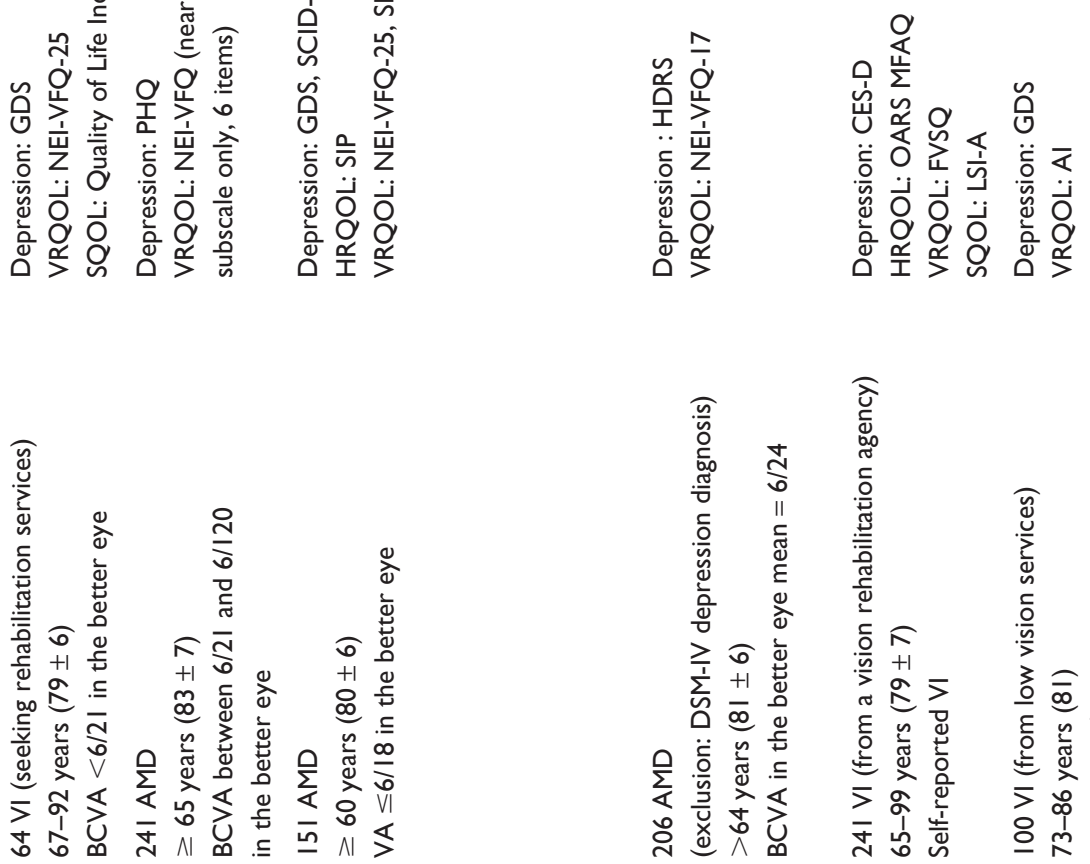

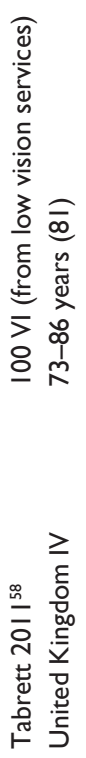

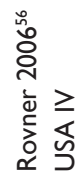

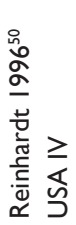


related QOL difference between the group with depressive symptomatology and the group without. In addition, Cohen's $d$ effect size values were medium to large, varying from 0.49 to 0.95 , with a median of 0.78 .

\section{Depression and vision-related QOL}

Vision-related QOL was assessed using five different instruments (number of studies): Activity Inventory ${ }^{51}(\mathrm{n}=1)$, Functional Vision Screening Questionnaire ${ }^{52}(n=5)$, National Eye Institute Visual Function Questionnaire ${ }^{53}$ (NEI-VFQ, $\mathrm{n}=4)$, Sickness Impact Profile specific to vision ${ }^{54}(\mathrm{n}=1)$, and Visual Functioning Index ${ }^{55}(\mathrm{n}=1)$.

Four of the five studies using the Functional Vision Screening Questionnaire found a significant association between more depressive symptoms and worse visionrelated QOL; three of these associations were weak ${ }^{37,40,50}$ while one was moderate. ${ }^{42}$ This last study, which included 51 older adults with age-related macular degeneration, revealed significantly poorer vision-related QOL for participants with significant depressive symptoms compared with nondepressed participants (medium effect size). ${ }^{42}$ In this same paper, variation in the depressive symptoms score was related to variation in the vision-related QOL score over a six-month period, and the incidence of significant depressive symptomatology was a significant determinant of decline in vision-related QOL. Further, participants who developed significant depressive symptoms at six months were 8.3 times more likely to experience a decline in their vision-related QOL than those who did not $(P=0.04) .{ }^{43}$ Conversely, a study of 584 elders with visual impairment seeking rehabilitation services did not find an association between depressive symptoms and vision-related QOL or any statistically significant difference in the Functional Vision Screening Questionnaire score between participants with major depression $(n=42)$, subthreshold depression $(n=157)$, or no depression $(n=385$, small effect size $) .{ }^{41}$

In the vision domain, the NEI-VFQ is one of the tools most widely used to assess vision-related QOL. In this review, three of the four studies using the NEI-VFQ concluded that a significant association exists between more severe depressive symptoms and worse vision-related QOL, ${ }^{14,45,56}$ while one found a trend. ${ }^{57}$ Two studies revealed a strong correlation ${ }^{14,45}$ and one found a weak correlation. ${ }^{56}$ The exclusion of individuals with depressive disorders might explain the weaker correlation in the last study. As well, the small percentage of elders with significant depressive symptoms (12.9\%) might explain why Rovner et al only found a tendency toward statistical significance in their cross-sectional study. ${ }^{57}$ The fact that they took into account only the near vision subscale of the NEI-VFQ, which contains items like reading ordinary print in newspapers or difficulty seeing well up close, such as when cooking or sewing, could be another explanation. Including more social and psychological subscales of the NEI-VFQ, such as social functioning, mental health, role difficulties, or dependency, could have resulted in a stronger and statistically significant correlation. In one of these studies, the group with depressive disorders (SCID-IV) had worse vision-related QOL than the group without depression. ${ }^{45}$ Further, Rovner et al indicated that participants with minimal depressive symptoms, who would not be considered as having significant depressive symptomatology according to usual standards, had worse vision-related QOL than nondepressed participants (medium effect size). ${ }^{56}$ After they controlled for age, gender, visual acuity, contrast sensitivity, and comorbidity, minimal depressive symptoms alone explained $4 \%$ of the variance in visionrelated QOL $(P=0.001)$.

In addition, Brody et al measured vision-related QOL using the vision-specific Sickness Impact Profile. Their results indicated that participants with depressive disorders (SCID-IV) had more vision disability than those without depression (medium effect size), and that more depressive symptoms were significantly related to greater vision disability (poorer vision-related QOL). ${ }^{45}$

One paper by Hayman et al evaluated the association between vision-related QOL, measured using the Visual Functioning Index, and depression in a sample of 391 elders with visual impairment in New Zealand. ${ }^{44}$ The results emphasized the difference in vision disability between participants with significant depressive symptomatology and nondepressed ones (medium effect size). The group with depressive symptoms presented greater visual disability but the two groups did not differ on visual acuity. ${ }^{44}$ This study also showed a significant association between fewer depressive symptoms and better vision-related QOL.

The Activity Inventory is another vision-related QOL questionnaire but in this case the individual has to rate the importance of each activity according to his/her lifestyle. Using that tool, Tabrett and Latham found that depressive symptoms had a strong correlation with vision-related QOL in a sample of 100 participants with recent visual impairment. ${ }^{58}$

In sum, nine studies ${ }^{14,37,40,42,44,45,50,56,58}$ showed a significant correlation between depressive symptoms and vision-related QOL; the correlation coefficients varied from 0.22 and 0.64 , with a median of 0.31 . One study found a trend $^{57}$ and one revealed no association. ${ }^{41}$ There was a 
vision-related QOL difference between the depressed group and the nondepressed group in five studies. ${ }^{41,42,44,45,56}$ The Cohen's $d$ were small to large, varying from 0.17 to 0.87 , with a median of 0.61 .

\section{Depression and subjective QOL}

In the vision domain, few studies have assessed subjective QOL in older adults. Only two papers in this review investigated the relationship between depressive symptoms and subjective QOL. ${ }^{14,50}$ One used the Life Satisfaction Index-A ${ }^{59}$ and the other used the Quality of Life Index. ${ }^{60}$

Reinhardt used the 18-item Life Satisfaction Index-A version ${ }^{61}$ to assess satisfaction with life in 241 older adults with visual impairment. ${ }^{50}$ She found a very strong association between fewer depressive symptoms and better subjective QOL.

The Quality of Life Index, frequently used with elders, assesses satisfaction with and the importance of different aspects of life. Satisfaction with life scores are weighted by importance scores. In the Canadian study using this instrument, depressive mood was strongly related with poorer subjective QOL in 64 elders with visual impairment seeking rehabilitation services. ${ }^{14}$ In that sample, depressive symptoms explained $45 \%$ of the subjective QOL score $(P<0.001)$.

In sum, the two studies showed a very strong significant correlation between depressive symptoms and subjective QOL, Pearson's $r$ being -0.68 and -0.72 .

\section{Discussion}

\section{Prevalence of depression}

As revealed by the reviewed studies, the prevalence of depressive mood in elders with visual impairment is high, ranging from $7 \%$ to $39 \%$ for clinical depression ${ }^{15,38,45,57}$ and from $29 \%$ to $43 \%$ for significant depressive symptoms. ${ }^{13,14,20,24,39,40,42,44}$ In comparison, it is estimated that $5 \%-8 \%$ of community-dwelling elders have depression, and somewhere between $13 \%$ and $27 \%$ have depressive symptoms. ${ }^{62-65}$ These troubling rates in older adults with visual impairment are often unsuspected or not well known in primary care and by vision professionals like ophthalmologists and optometrists. Although the high prevalence of depression is more likely to be recognized by low vision rehabilitation specialists, they often do not address this important mental disorder or offer the client appropriate treatment for it. Yet depressive symptoms can have significant effects. Depressed elders left without proper treatment may experience serious consequences, including increased disability, malnutrition, institutionalization, and even mortality. ${ }^{10,66-71} \mathrm{In}$ addition, depressed elders tend to underestimate their functional capacities ${ }^{72,73}$ leading to activity restrictions. This decrease in perceived functional status also seems to be present in the older adults with visual impairment. In fact, six studies found that older adults with visual impairment who had depression disorders, significant depressive symptoms, or even minimal depressive symptoms had significantly lower health-related and vision-related QOL than nondepressed elders with visual impairment. . $^{24,41,42,44,45,56}$

\section{Depression, and health-related and vision-related $\mathrm{QOL}$}

Nearly all of the articles examined in this review revealed that lower health-related or vision-related QOL was associated with greater symptoms of depression. This significant relationship between depressive symptoms and healthrelated or vision-related QOL is not surprising. The effect of depression on functional disability and vice versa has been well established for community-dwelling elders. ${ }^{66,74-76}$ In a community-based study including almost 7000 participants, depression was positively associated with four times more risk of disability. ${ }^{77}$ Also, a six-year prospective study of community-dwelling depressed elders found an increase in functional limitation over time. ${ }^{78}$ Conversely, previous studies demonstrated that a reduction in function increases the risk of depressive mood. ${ }^{79}$

Most studies in this review had a cross-sectional design and therefore could not determine if depression caused an increase in perceived functional disability or if functional disability led to depression. In other words, the change in perceived function is related to increased depressive symptoms, but a cause-and-effect relationship has not yet been established in individuals with visual impairment. However, one longitudinal study of older adults with visual impairment newly enrolled for low vision services showed that greater disability at baseline was a significant predictor of depressive symptoms at six months, after controlling for baseline depressive symptoms and sociodemographic factors, while the opposite was not true. ${ }^{41}$ This suggests that disability would be a risk factor for depression in this population. On the other hand, incident depressive symptoms were a significant correlate of vision-related QOL decline in a sixmonth follow-up study of elders with age-related macular degeneration. ${ }^{42}$ Now that the significant correlation between these two variables has been clearly described, more research is needed to assess which comes first and to pinpoint the determinants and mediators of this relationship. For the majority of studies, the impact of depressive symptoms on QOL was not their purpose, so almost no attempt was made to 
explain the relationship between those two variables. On the other hand, Rovner et al were specifically interested in that relationship and found that the personality trait of neuroticism was a risk factor for depression. More studies are essential to understand better the relationship between depression and QOL of elders with visual impairment.

It is important to mention that a dichotomy can exist between self-reported measures and objective testing. In clinical settings, this reality is particularly important because the objective performance of a person with visual impairment (eg, being able to read newspaper print, having good reading speed) is not a guarantee that the person is happy with his/her vision or has good QOL. In fact, some studies in this review showed worse vision-related QOL for depressed elders with visual impairment compared with their nondepressed counterparts, but no significant difference in visual acuity between the two groups. ${ }^{24,44,56}$ Moreover, in a follow-up study of older adults with newly bilateral agerelated macular degeneration, a decrease in vision-related QOL (perceived functional vision) was significantly associated with an increase in depressive symptoms, independent of a change in visual acuity (visual function). ${ }^{42}$ Some authors also maintain that the discrepancy between subjective difficulties and objective function could be a warning sign of depression because individuals with depression tend to underestimate their executive performance and have lower QOL. ${ }^{12,73,80}$

\section{Depression and subjective QOL}

While health-related and vision-related QOL questionnaires address mainly performance, subjective QOL approaches try to understand the more comprehensive aspects of QOL. ${ }^{17}$ The two studies looking at the association between depressive symptoms and subjective QOL highlighted strong correlations. ${ }^{14,50}$ In one of these studies, almost half of the subjective QOL was explained by depressive symptoms alone. ${ }^{14}$ Since depression and subjective QOL both rely on feelings or perceptions, there might be an overlap that explains the strong correlation found. It has already been suggested that these two concepts, depression and subjective QOL, are somewhat similar.

So far we have looked at the relationship between QOL and depression in elders. Fortunately, treatment of depression by medication and psychotherapy in community-dwelling elders has been shown to improve perceived function. ${ }^{81,82}$ Also, studies which examined treatment of depression by self-management or problem-solving programs have shown that focusing on ways to deal with life differently can help to decrease emotional distress and increase QOL. ${ }^{27,83,84}$ There is also evidence that self-management programs ${ }^{85-89}$ and pharmacological treatment ${ }^{90}$ can reduce depressive symptoms in elders with visual impairment. Studies also showed the feasibility of delivering self-management or problemsolving programs in usual vision service facilities. ${ }^{85,87,91,92}$ Implementing such programs in vision clinics and low vision rehabilitation centers could be beneficial for elders with visual impairment. However, further research is needed to evaluate the long-term effects of such interventions and whether pharmacological treatment should be given in conjunction with psychological services.

In order to treat depression, first it has to be diagnosed. As already mentioned, late-life depression in individuals with visual impairment is often unrecognized and untreated. ${ }^{93}$ It is not just older adults with visual impairment who are undiagnosed but the elderly population in general. ${ }^{94-97}$ Some efforts have been made to implement collaborative depression management models for the elderly ${ }^{98-100}$ but there is still a lot to do. Unless the patient directly tells the clinician that he/ she has depressive symptoms, the professional has to search for the signs and symptoms. However, it is easier to blame the ocular disease for poor reading performance than to look for depression. Also, it requires trained professionals who know what to look for in depression. ${ }^{101}$ In addition, referring patients to the right services and defining professional/ personal responsibilities are among the problems to be resolved..$^{93}$ A program addressing depression issues with health care professionals, with the goal of improving the detection and management of depressive elders with visual impairment, has been developed. ${ }^{102}$ A lot of sensitization remains to be done in geriatric services.

\section{Conclusion}

This review highlighted the association between more severe depressive symptoms and worse QOL in older adults with visual impairment. Additional studies are necessary to pinpoint further the determinants and mediators of this relationship. Considering the high prevalence of depression in this community and its disabling effects on QOL, interventions to prevent and treat depression are essential. More efforts are needed in clinical settings to train health care practitioners to identify depressed elders with visual impairment and provide appropriate treatment.

\section{Disclosure}

The authors report no conflicts of interest in this work. 


\section{References}

1. Wallhagen MI, Strawbridge WJ, Shema SJ, Kurata J, Kaplan GA. Comparative impact of hearing and vision impairment on subsequent functioning. J Am Geriatr Soc. 2001;49(8):1086-1092.

2. The Lighthouse Inc. The Lighthouse National Survey on Vision Loss: the experience, attitudes, and knowledge of middle-aged and older Americans. 1995. Available from: http://www.lighthouse.org/research/ archived-studies/national-survey/. Accessed August 1, 2012.

3. Crews JE, Campbell VA. Health conditions, activity limitations, and participation restrictions among older people with visual impairment. J Vis Impair Blind. 2001;95(8):453-467.

4. Horowitz AB, Reinhardt M, Joann P. Prevalence and risk factors for self-reported visual impairment among middle-aged and older adults. Res Aging. 2005;27:307-326.

5. Desrosiers J, Wanet-Defalque MC, Temisjian K, et al. Participation in daily activities and social roles of older adults with visual impairment. Disabil Rehabil. 2009;31(15):1227-1234.

6. Alma MA, van der Mei SF, Melis-Dankers BJ, van Tilburg TG, Groothoff JW, Suurmeijer TP. Participation of the elderly after vision loss. Disabil Rehabil. 2011;33(1):63-72.

7. Cook G, Brown-Wilson C, Forte D. The impact of sensory impairment on social interaction between residents in care homes. Int J Older People Nurs. 2006;1(4):216-224.

8. Defini J, Burack-Weiss A. Psychosocial assessment of adults with vision impairments. In: Silverstone B, Lang MA, Rosenthal BP, Fayes E, editors. The Lighthouse Handbook on Vision Impairment and Vision Rehabilitation. Volume 2. New York, NY: Oxford University Press; 2000.

9. Cabrera MA, Mesas AE, Garcia AR, de Andrade SM. Malnutrition and depression among community-dwelling elderly people. J Am Med Dir Assoc. 2007;8(9):582-584.

10. Yoshimura K, Yamada M, Kajiwara Y, Nishiguchi S, Aoyama T. Relationship between depression and risk of malnutrition among community-dwelling young-old and old-old elderly people. Aging Ment Health. 2013;17(4):456-460.

11. Verstraten PFJ, Brinkmann WLJH, Stevens NL, Schouten JSAG. Loneliness, adaptation to vision impairment, social support and depression among visually impaired elderly. Int Congr Ser. 2005;1282(0): 317-321.

12. Casten R, Rovner B. Depression in age-related macular degeneration. J Vis Impair Blind. 2008;102(10):591-599.

13. Mathew RS, Delbaere K, Lord SR, Beaumont P, Vaegan, Madigan MC. Depressive symptoms and quality of life in people with age-related macular degeneration. Ophthalmic Physiol Opt. 2011;31(4): $375-380$.

14. Renaud J, Levasseur M, Gresset J, et al. Health-related and subjective quality of life of older adults with visual impairment. Disabil Rehabil. 2010;32(11):899-907.

15. Horowitz A, Reinhardt JP, Kennedy GJ. Major and subthreshold depression among older adults seeking vision rehabilitation services. Am J Geriatr Psychiatry. 2005;13(3):180-187.

16. Dijkers MP. Quality of life of individuals with spinal cord injury: a review of conceptualization, measurement, and research findings. J Rehabil Res Dev. 2005;42(3 Suppl 1):87-110.

17. Haas BK. Clarification and integration of similar quality of life concepts. Image J Nurs Sch. 1999;31(3):215-220.

18. Levasseur M, Tribble DS-C, Desrosiers J. Analysis of quality of life concept in the context of older adults with physical disabilities. Can J Occup Ther. 2006;73(3):163-177. French.

19. Tielsch JM, Sommer A, Witt K, Katz J, Royall RM. Blindness and visual impairment in an American urban population: the Baltimore Eye Survey. Arch Ophthalmol. 1990;108(2):286-290.

20. Reinhardt JP. Effects of positive and negative support received and provided on adaptation to chronic visual impairment. Appl Dev Sci. 2001;5(2):76-85.

21. Hooper P, Jutai JW, Strong G, Russell-Minda E. Age-related macular degeneration and low-vision rehabilitation: a systematic review. Can J Ophthalmol. 2008;43(2):180-187.
22. Brown GC, Brown MM, Sharma S, et al. The burden of age-related macular degeneration: a value-based medicine analysis. Trans Am Ophthalmol Soc. 2005;103:173-184.

23. Silverstone B, Lang M, Rosenthal B, Faye EE. The Lighthouse Handbook on Vision Impairment and Vision Rehabilitation. Volume II. New York, NY: Oxford University Press; 2000.

24. Rovner BW, Zisselman PM, Shmuely-Dulitzki Y. Depression and disability in older people with impaired vision: a follow-up study. $\mathrm{JAm}$ Geriatr Soc. 1996;44(2):181-184.

25. Evans JR, Fletcher AE, Wormald RP. Depression and anxiety in visually impaired older people. Ophthalmology. 2007;114(2):283-288.

26. Burmedi D, Becker S, Heyl V, Wahl H-W, Himmelsbach I. Emotional and social consequences of age-related low vision. Vis Impair Res. 2002;4(1):47.

27. Lamers F, Jonkers CC, Bosma H, Diederiks JP, van Eijk JT. Effectiveness and cost-effectiveness of a minimal psychological intervention to reduce non-severe depression in chronically ill elderly patients: the design of a randomised controlled trial [ISRCTN92331982]. BMC Public Health. 2006;6:161.

28. IsHak WW, Greenberg JM, Balayan K, et al. Quality of life: the ultimate outcome measure of interventions in major depressive disorder. Harv Rev Psychiatry. 2011;19(5):229-239.

29. National Institute of Mental Health. Depression. Department of Health and Human Services; 2011. Available from: http://www.nimh.nih. gov/health/publications/depression/depression-booklet.pdf. Accessed November 8, 2012.

30. Verbrugge LM, Jette AM. The disablement process. Soc Sci Med. 1994;38(1):1-14.

31. West S, Sommer A. Prevention of blindness and priorities for the future. Bull World Health Organ. 2001;79(3):2.

32. Taylor HR, Keeffe JE, Vu HT, et al. Vision loss in Australia. Med J Aust. 2005;182(11):565-568.

33. Sanderson S, Tatt ID, Higgins JP. Tools for assessing quality and susceptibility to bias in observational studies in epidemiology: a systematic review and annotated bibliography. Int J Epidemiol. 2007;36(3): 666-676.

34. Cho MK, Bero LA. Instruments for assessing the quality of drug studies published in the medical literature. JAMA. 1994;272(2):101-104.

35. Bergner M, Bobbitt RA, Carter WB, Gilson BS. The Sickness Impact Profile: development and final revision of a health status measure. Med Care. 1981;19(8):787-805.

36. Cohen J. Statistical Power Analysis for the Behavioral Sciences. 2nd ed. Hillsdale, NJ: L Erlbaum Associates; 1988.

37. Reinhardt JP, Boerner K, Horowitz A. Personal and social resources and adaptation to chronic vision impairment over time. Aging Ment Health. 2009;13(3):367-375.

38. Shmuely-Dulitzki Y, Rovner BW, Zisselman P. The impact of depression on functioning in elderly patients with low vision. $A m J$ Geriatr Psychiatry. 1995;3(4):325-329.

39. Horowitz A, Reinhardt J, Boerner K, Travis L. The influence of health, social support quality and rehabilitation on depression among disabled elders. Aging Ment Health. 2003;7(5):342-350.

40. Horowitz A, Reinhardt JP, Boerner K. The effect of rehabilitation on depression among visually disabled older adults. Aging Ment Health. 2005;9(6):563-570.

41. Horowitz A, Brennan M, Reinhardt JP, MacMillan T. The impact of assistive device use on disability and depression among older adults with age-related vision impairments. J Gerontol B Psychol Sci Soc Sci. 2006;61B(5):S274-S280.

42. Rovner BW, Casten RJ, Tasman WS. Effect of depression on vision function in age-related macular degeneration. Arch Ophthalmol. 2002;120(8):1041-1044.

43. Rovner BW, Casten RJ. Neuroticism predicts depression and disability in age-related macular degeneration. J Am Geriatr Soc. 2001;49(8): $1097-1100$.

44. Hayman KJ, Kerse NM, La Grow SJ, Wouldes T, Robertson MC, Campbell AJ. Depression in older people: visual impairment and subjective ratings of health. Optom Vis Sci. 2007;84(11):1024-1030. 
45. Brody BL, Gamst AC, Williams RA, et al. Depression, visual acuity, comorbidity, and disability associated with age-related macular degeneration. Ophthalmology. 2001;108(10):1893-1900.

46. Folstein MF, Romanoski AJ, Nestadt G, et al. Brief report on the clinical reappraisal of the Diagnostic Interview Schedule carried out at the Johns Hopkins site of the Epidemiological Catchment Area Program of the NIMH. Psychol Med. 1985;15(4):809-814.

47. Ware JE Jr, Sherbourne CD. The MOS 36-item Short-Form Health Survey (SF-36). I. Conceptual framework and item selection. Med Care. 1992;30(6):473-483.

48. Center for the Study of Aging and Human Development. The OARS Methodology. 1st ed. Durham, NC: Duke University; 1975.

49. Bergner M, Bobbitt RA, Kressel S, Pollard WE, Gilson BS, Morris JR. The Sickness Impact Profile: conceptual formulation and methodology for the development of a health status measure. Int J Health Serv. 1976;6(3):393-415.

50. Reinhardt JP. The importance of friendship and family support in adaptation to chronic vision impairment. J Gerontol B Psychol Sci Soc Sci. 1996;51B(5):P268-P278.

51. Massof RW, Ahmadian L, Grover LL, et al. The Activity Inventory: an adaptive visual function questionnaire. Optom Vis Sci. 2007;84(8): 763-774.

52. Horowitz A, Teresi JA, Cassels LA. Development of a vision screening questionnaire for older people. J Gerontol Soc Work. 1991;17(3-4): 37-56.

53. Mangione CM, Lee PP, Pitts J, Gutierrez P, Berry S, Hays RD. Psychometric properties of the National Eye Institute Visual Function Questionnaire (NEI-VFQ). NEI-VFQ Field Test Investigators. Arch Ophthalmol. 1998;116(11):1496-1504.

54. Scott IU, Schein OD, West S, Bandeen-Roche K, Enger C, Folstein MF. Functional status and quality of life measurement among ophthalmic patients. Arch Ophthalmol. 1994;112(3):329-335.

55. Steinberg EP, Tielsch JM, Schein OD, et al. The VF-14. An index of functional impairment in patients with cataract. Arch Ophthalmol. 1994;112(5):630-638.

56. Rovner BW, Casten RJ, Hegel MT, Tasman WS. Minimal depression and vision function in age-related macular degeneration. Ophthalmology. 2006;113(10):1743-1747.

57. Rovner BW, Casten RJ, Massof RW, Leiby BE, Tasman WS; Wills Eye AMD Study. Psychological and cognitive determinants of vision function in age-related macular degeneration. Arch Ophthalmol. 2011;129(7):885-890.

58. Tabrett DR, Latham K. Factors influencing self-reported vision-related activity limitation in the visually impaired. Invest Ophthalmol Vis Sci. 2011;52(8):5293-5302.

59. Neugarten BL, Havighurst RJ, Tobin SS. The measurement of life satisfaction. J Gerontol. 1961;16:134-143.

60. Ferrans CE, Powers MJ. Quality of life index: development and psychometric properties. ANS Adv Nurs Sci. 1985;8(1):15-24.

61. Adams DL. Analysis of a life satisfaction index. J Gerontol. 1969;24(4): 470-474.

62. Blazer D, Hughes DC, George LK. The epidemiology of depression in an elderly community population. Gerontologist. 1987;27(3):281-287.

63. Grunebaum MF, Oquendo MA, Manly JJ. Depressive symptoms and antidepressant use in a random community sample of ethnically diverse, urban elder persons. J Affect Disord. 2008;105(1-3):273-277.

64. Park JH, Lee JJ, Lee SB, et al. Prevalence of major depressive disorder and minor depressive disorder in an elderly Korean population: results from the Korean Longitudinal Study on Health and Aging (KLoSHA). $J$ Affect Disord. 2010;125(1-3):234-240.

65. Futterman A, Thompson L, Gallagher-Thompson D, Ferris R. Depression in later life: epidemiology, assessment, etiology, and treatment. Handbook of Depression. 2nd ed. New York, NY: Guilford Press; 1995.

66. Bruce ML, Seeman TE, Merrill SS, Blazer DG. The impact of depressive symptomatology on physical disability: MacArthur studies of successful aging. Am J Public Health. 1994;84(11):1796-1799.
67. Katon WJ. Clinical and health services relationships between major depression, depressive symptoms, and general medical illness. Biol Psychiatry. 2003;54(3):216-226.

68. Young BA, Von Korff M, Heckbert SR, et al. Association of major depression and mortality in Stage 5 diabetic chronic kidney disease. Gen Hosp Psychiatry. 2010;32(2):119-124.

69. Cullum S, Metcalfe C, Todd C, Brayne C. Does depression predict adverse outcomes for older medical inpatients? A prospective cohort study of individuals screened for a trial. Age Ageing. 2008;37(6): 690-695.

70. Leng CH, Wang JD. Long term determinants of functional decline of mobility: an 11-year follow-up of 5464 adults of late middle aged and elderly. Arch Gerontol Geriatr. 2013;57(2):215-220.

71. Kaburagi T, Hirasawa R, Yoshino H, et al. Nutritional status is strongly correlated with grip strength and depression in community-living elderly Japanese. Public Health Nutr. 2011;14(11):1893-1899.

72. Kiyak HA, Teri L, Borson S. Physical and functional health assessment in normal aging and in Alzheimer's disease: self-reports vs family reports. Gerontologist. 1994;34(3):324-330.

73. Kuriansky JB, Gurland BJ, Fleiss JL. The assessment of self-care capacity in geriatric psychiatric patients by objective and subjective methods. J Clin Psychol. 1976;32(1):95-102.

74. Kennedy GJ, Kelman HR, Thomas C. The emergence of depressive symptoms in late life: the importance of declining health and increasing disability. J Community Health. 1990;15(2):93-104.

75. Koenig HG, George LK. Depression and physical disability outcomes in depressed medically ill hospitalized older adults. Am J Geriatr Psychiatry. 1998;6(3):230-247.

76. Penninx BW, Guralnik JM, Ferrucci L, Simonsick EM, Deeg DJ, Wallace RB. Depressive symptoms and physical decline in communitydwelling older persons. JAMA. 1998;279(21):1720-1726.

77. Dunlop DD, Manheim LM, Song J, Lyons JS, Chang RW. Incidence of disability among preretirement adults: the impact of depression. $\mathrm{Am}$ J Public Health. 2005;95(11):2003-2008.

78. Cronin-Stubbs D, de Leon CF, Beckett LA, Field TS, Glynn RJ, Evans DA. Six-year effect of depressive symptoms on the course of physical disability in community-living older adults. Arch Intern Med. 2000;160(20):3074-3080.

79. Barry LC, Soulos PR, Murphy TE, Kasl SV, Gill TM. Association between indicators of disability burden and subsequent depression among older persons. J Gerontol A Biol Sci Med Sci. 2013;68(3): 286-292.

80. Blazer DG. Depression in late life: review and commentary. J Gerontol A Biol Sci Med Sci. 2003;58A(3):249-265.

81. Katon WJ. Epidemiology and treatment of depression in patients with chronic medical illness. Dialogues Clin Neurosci. 2011;13(1): 7-23.

82. Ishak WW, Ha K, Kapitanski N, et al. The impact of psychotherapy, pharmacotherapy, and their combination on quality of life in depression. Harv Rev Psychiatry. 2011;19(6):277-289.

83. Areán PA, Raue P, Mackin RS, Kanellopoulos D, McCulloch C, Alexopoulos GS. Problem-solving therapy and supportive therapy in older adults with major depression and executive dysfunction. Am J Psychiatry. 2010;167(11):1391-1398.

84. Tousman S, Zeitz H, Taylor LD. A pilot study assessing the impact of a learner-centered adult asthma self-management program on psychological outcomes. Clin Nurs Res. 2010;19(1):71-88.

85. Girdler SJ, Boldy DP, Dhaliwal SS, Crowley M, Packer TL. Vision self-management for older adults: a randomised controlled trial. $\mathrm{Br} J$ Ophthalmol. 2010;94(2):223-228.

86. Packer TL, Girdler S, Boldy DP, Dhaliwal SS, Crowley M. Vision self-management for older adults: a pilot study. Disabil Rehabil. 2009;31(16):1353-1361.

87. Brody BL, Roch-Levecq A-C, Gamst AC, Maclean K, Kaplan RM, Brown SI. Self-management of age-related macular degeneration and quality of life: a randomized controlled trial. Arch Ophthalmol. 2002;120(11):1477-1483. 
88. Brody BL, Roch-Levecq A-C, Thomas RG, Kaplan RM, Brown SI. Self-management of age-related macular degeneration at the 6-month follow-up: a randomized controlled trial. Arch Ophthalmol. 2005;123(1): 46-53.

89. Lee L, Packer TL, Tang SH, Girdler S. Self-management education programs for age-related macular degeneration: a systematic review. Australas J Ageing. 2008;27(4):170-176.

90. Brody BL, Field LC, Roch-Levecq A-C, Moutier CY, Edland SD, Brown SI. Treatment of depression associated with age-related macular degeneration: a double-blind, randomized, controlled study. Ann Clin Psychiatry. 2011;23(4):277-284.

91. Rovner BW, Casten RJ, Hegel MT, Leiby BE, Tasman WS. Preventing depression in age-related macular degeneration. Arch Gen Psychiatry. 2007;64(8):886-892.

92. Packer TL, Girdler S, Boldy DP, Dhaliwal SS, Crowley M. Vision self-management for older adults: a pilot study. Disabil Rehabil. 2009;31(16):1353-1361.

93. Fenwick EK, Lamoureux EL, Keeffe JE, Mellor D, Rees G. Detection and management of depression in patients with vision impairment. Optom Vis Sci. 2009;86(8):948-954.

94. Unutzer J. Diagnosis and treatment of older adults with depression in primary care. Biol Psychiatry. 2002;52(3):285-292.

95. Baker J, Keenan L, Zwischenberger J. A model for primary care psychology with general thoracic surgical patients. J Clin Psychol Med Settings. 2005;12(4):359-366.
96. Saunders SM, Wojcik JV. The reliability and validity of a brief self-report questionnaire to screen for mental health problems: the Health Dynamics Inventory. J Clin Psychol Med Settings. 2004;11(3):233-241.

97. Crystal S, Sambamoorthi U, Walkup JT, Akincigil A. Diagnosis and treatment of depression in the elderly Medicare population: predictors, disparities, and trends. J Am Geriatr Soc. 2003;51(12):1718-1728.

98. Unutzer J, Katon W, Callahan CM, et al. Collaborative care management of late-life depression in the primary care setting: a randomized controlled trial. JAMA. 2002;288(22):2836-2845.

99. Beekman AT, van der Feltz-Cornelis C, van Marwijk HW. Enhanced care for depression. Curr Opin Psychiatry. 2013;26(1):7-12.

100. Simon G. Collaborative care for mood disorders. Curr Opin Psychiatry. 2009;22(1):37-41.

101. Haley WE, McDaniel SH, Bray JH, et al. Psychological practice in primary care settings: practical tips for clinicians. Prof Psychol Res Pr. 1998;29(3):237-244.

102. Rees G, Mellor D, Heenan M, et al. Depression training program for eye health and rehabilitation professionals. Optom Vis Sci. 2010;87(7):494-500.
Clinical Interventions in Aging

\section{Publish your work in this journal}

Clinical Interventions in Aging is an international, peer-reviewed journal focusing on evidence-based reports on the value or lack thereof of treatments intended to prevent or delay the onset of maladaptive correlates of aging in human beings. This journal is indexed on PubMed Central, MedLine, the American Chemical Society's 'Chemical Abstracts

\section{Dovepress}

Service' (CAS), Scopus and the Elsevier Bibliographic databases. The manuscript management system is completely online and includes a very quick and fair peer-review system, which is all easy to use. Visit http://www.dovepress.com/testimonials.php to read real quotes from published authors. 